\section{Development of in vitro 3D culture system to mimic lung cancer tissue}

\author{
Désirée Baruffaldi, 1,2 Marta Canta,1 \\ Candido Fabrizio Pirri,1-3 \\ Francesca Frascella1,2
}

1Dipartimento di Scienza Applicata e Tecnologia, Politecnico di Torino, Turin; 2PolitoBIOMed Lab, Politecnico di Torino, Turin; ${ }^{3}$ Center for Sustainable Future Technologies @Polito, Istituto Italiano di Tecnologia, Turin, Italy

\begin{abstract}
A 3D culture system based on a photocurable matrix has been developed. The aim is to create a $3 \mathrm{D}$ printable platform mimicking lung cancer tissue, to study tumor microenvironment evolution, in terms of structural (architecture) and molecular (signalling) components.
\end{abstract}

\section{Introduction}

Non-small cell lung cancers (NSCLCs) represent about the $80 \%$ to $85 \%$ of total lung cancers and consist in several subtypes including adenocarcinoma that derives from cells that normally secrete mucus. ${ }^{1}$ It is well known that cancer progression is promoted by the tumor microenvironment, which is made of several cell types (e.g. fibroblasts, immune and endothelial cells), matrix components (e.g. collagen and fibronectin) and signaling molecules. ${ }^{2}$ In addition, another important player is angiogenesis which sustains cancer growth and promotes metastasis. ${ }^{3}$ The aim of the study is to mimic the tumor microenvironment by culturing tumor cells and fibroblasts in a 3D matrix, made of methacrylated gelatin (GelMA). Particularly, cell--cell and cell-matrix interactions will be analyzed, focusing on how these change the surrounding matrix in terms of composition and stiffness. Then, channels will be added to the 3D model, by using a 3D bioprinter, to reproduce physiological vasculature. This improvement will be useful to better study how endothelial cells, which adhere to channel's wall, and continuous nutrient flow affect tumor cell behavior and matrix properties.

\section{Materials and Methods}

Synthesis of GelMA derived from a protocol previously described. ${ }^{4}$ In particular, 10\%(w/v) type B gelatin from bovine skin (Sigma Aldrich) was dissolved into Dulbecco's Phosphate Buffered Saline (DPBS, Sigma) at $50^{\circ} \mathrm{C}$. Methacrylic Anhydride (MA, Sigma) was slowly added and the reaction lasted 2 hours. Then, the solution was diluted and dialyzed with cellulose membrane (Sigma) for 1 week. Finally, GelMA was freeze-dried. For 3D cell culture, lung cancer epithelial cell lines, A549, and normal lung fibroblast MRC-5 were mixed with GelMA at the density of $1,5^{*} 10^{6}$ cells $/ \mathrm{mL}$. For proliferation experiment, 50 ul of cell-loaded GelMA were photopolymerized into 96-well for 2 minutes with a $405 \mathrm{~nm}$ LED. Then, MTT (Sigma) assay was performed after 1 day or 6 days of culturing.

\section{Results}

Previous works described the possibility to use GelMA as matrix for cell culture.5,6 Since the goal of the study is to create a system that mimics the tumor microenvironment, the 3D hydrogel model has been loaded with both tumor cells (A549) and fibroblasts (MRC-5) at different ratios (1:1 and 1:2). Taking into account that matrix stiffness strongly impacts on cell behavior, ${ }^{7}$ two different GelMA formulations (Medium and High) have been used, characterized by different degrees of substitution. Then, to test the proliferation, MTT assay was performed at various time points. As shown in Figure 1, cells grown both in monoculture and in coculture systems suggesting that the two cell lines could be used together for further experiments. Moreover, the presence of tumor cells enhanced fibroblasts viability suggesting the presence of a crosstalk between the two cell types. Finally, not
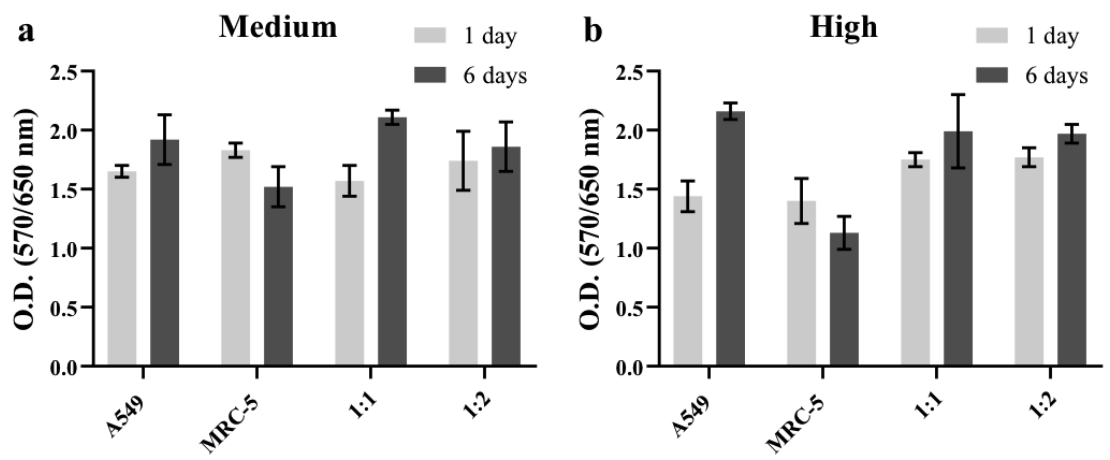

Figure 1. MTT assay. A549 and MRC-5 were loaded into GelMA Medium (a) and High (b) at the density of $1,5 \times 10^{6} \mathrm{cell} / \mathrm{s} / \mathrm{ml}$ as monoculture or co-culture (1:1 and 1:2).
Correspondence:Désirée Baruffaldi, Dipartimento di Scienza Applicata e Tecnologia, Politecnico di Torino, C.so Duca degli Abruzzi 24, Turin 10129, Italy.

E-mail: desiree.baruffaldi@polito.it

Key words: Lung adenocarcinoma; Extracellular Matrix (ECM); Bioprinting.

Acknowledgments: We thank Dr. Valentina Monica for providing us A549 cells and Prof. Chiara Tondaturo for MRC-5 cells.

Disclosures: The authors declare that they have no known conflict of interest.

Conference presentation: This paper was presented at the Third Centro 3R Annual Meeting - L'era delle 3R: modelli in silico, in vitro e in vivo per promuovere la ricerca traslazionale 30 September - 1 October 2021, Evento online organizzato dal Politecnico di Torino.

Received for publication: 9 July 2021.

Accepted for publication: 7 September 2021.

This work is licensed under a Creative Commons Attribution NonCommercial 4.0 License (CC BY-NC 4.0).

(C) Copyright: the Author(s), 2021

Licensee PAGEPress, Italy

Biomedical Science and Engineering 2021; 4(s1):149 doi:10.4081/bse.2021.149

strongly differences in proliferation were detected between two degrees of methacrylation. Next, we will assess how tumor cells influence fibroblast behavior in terms of production of matrix components (e.g., fibronectin and collagen) and of changes in matrix stiffness. Finally, the microfluidic devices will be obtained with a 3D bioprinter and the role of endothelial cells in matrix modification will be analyzed. 


\section{Discussion and Conclusions}

Our findings suggest that 3D system of lung cancer could be obtained by using a photosensitive matrix that does not affect cell viability. $3 \mathrm{D}$ co-culture better mimics the real tumor microenvironment and allows the dissection of the role of different cell types in cancer progression. Moreover, the introduction of microfluidic system resembles the normal nutrient flow and could be implemented as preferable system for drugs testing.

\section{References}

1. American Cancer Society. What is lung cancer? Available from: https://www.cancer.org/cancer/lungcancer/about/what-is.html

2. Joyce JA, Pollard JW. Microenvironmental regulation of metastasis. Nat Rev Cancer 2009;9: 239-52.

3. Nishida N, Yano $H$, Nishida $T$, et al. Angiogenesis in cancer. Vasc Health Risk Manag 2006;2:213-9.

4. Nichol JW, Koshy ST, Bae H, et al.
Cell-laden microengineered gelatin methacrylate hydrogels. Biomaterials 2010;31:5536-44.

5. Bertassoni LE, Cardoso JC, Manoharan $\mathrm{V}$, et al. Direct-write bioprinting of cellladen methacrylated gelatin hydrogels. Biofabrication 2014;6:024105.

6. Kolesky DB, Truby RL, Gladman AS, et al. 3D bioprinting of vascularized, heterogeneous cell-laden tissue constructs. Adv Mater 2014;26:3124-30.

7. Baruffaldi D, Palmara G, Pirri C, Frascella F. 3D Cell Culture: Recent Development in Materials with Tunable Stiffness. ACS Appl Bio Mater 2021;4:2233-50. 